\section{L'Actualité économique}

L'ACTUALITÉ ÉCONOMIQUE

\section{De la neutralité de la monnaie}

\section{Yves Rabeau}

Volume 44, numéro 1, avril-juin 1968

URI : https://id.erudit.org/iderudit/1002998ar

DOI : https://doi.org/10.7202/1002998ar

Aller au sommaire du numéro

Éditeur(s)

HEC Montréal

ISSN

0001-771X (imprimé)

1710-3991 (numérique)

Découvrir la revue

Citer cet article

Rabeau, Y. (1968). De la neutralité de la monnaie. L'Actualité économique, 44(1), 43-63. https://doi.org/10.7202/1002998ar d'utilisation que vous pouvez consulter en ligne.

https://apropos.erudit.org/fr/usagers/politique-dutilisation/ 


\section{De la neutralité de la monnaie*}

La neutralité de la monnaie n'est pas un sujet nouveau. Elle retient encore une fois lattention dans ses rapports avec la croissance économique. Toutefois, ce n'est plus à l'effet d'un accrois' sement donné de la masse monétaire qu'on s'intéresse, mais aux conséquences d'une variation du rythme d'expansion de la masse monétaire. Cela relève de la dynamique.

Juste avant l'intérêt suscité par les modèles de croissance, la querelle provoquée par Patinkin sur la neutralité de la monnaie avait fait apparaitre une littérature considérable sur ces chapitres de la théorie économique, entre autres, louvrage de Gurley et Shaw, Money in a Theory of Finance. Dans la nouvelle édition de son célèbre volume, Don Patinkin a repris et synthétisé les diffé rentes critiques qu'on lui avait adressées et il semble qu'il soit possible maintenant d'arriver à un nombre de propositions qui couvrent assez bien le domaine statique.

Nous nous proposons de présenter, sous forme de théorèmes, différentes conclusions que certains auteurs ont formulées sur la question analysée en statique. Et dans la deuxième partie de l'ar' ticle, nous donnerons une synthèse sommaire de récents articles publiés dans le domaine de la croissance; les travaux ayant trait à la monnaie et à la croissance revêtent un caractère moins définitif que les questions statiques et nous avons l'intention de mettre en relief l'aspect tentative initiale de ces recherches qui devront éventuellement être poursuivies ${ }^{1}$.

- L'auteur est étudiant au doctorat au Massachusetts Institute of Technology et a bénéficié des cours des professeurs P. Samuelson, R. Solow, M. Sidrauski et G. Sparks pour écrire cet article.

1. Dans le texte qui suit, un chiffre entre crochets renvoie à la bibliographie donnée à la fin de l'article. Un chiffre entre parenthèses se rapporte à une équation. 
Nous allons débuter par la méthode classique où l'on suppose. la parfaite flexibilité des prix nominaux et des salaires. De plus, nous ferons les hypothèses suivantes : certitude; absence d'illusion monétaire (nous préférons utiliser immédiatement la définition que Patinkin donne de ce concept de sorte que nous laissons de côté, au départ, la querelle sur le postulat d'homogénéité en prenant pour acquis que les excès de demande des individus ne sont pas insen. sibles à une variation des prix monétaires, mais restent intouchés par un changement (évidemment équi-proportionnel) dans les prix de compte) ; élasticité d'anticipation égale à l'unité ; absence des effets de répartition.

Nous pouvons facilement déduire le revenu national de plein emploi par les équations traditionnelles suivantes:

$$
Q=F\left(K_{0}, L\right)
$$

soit la fonction de production macróéconomique ( $Q$ est l'output; $K$ le stock de capital constant à court terme et $L$, la main-d'œuvre).

L'offre de travail $\left(L_{s}\right)$ dépend du salaire réel ( $w=$ salaire nominal, $P$, niveau général des prix) et la demande de main-d'œuvre $\left(L_{d}\right)$ est la productivité marginale de la main-d'œuvre:

$$
L_{s}=L\left(\frac{w}{P}\right)
$$

A l'équilibre, on retrouve :

$$
\frac{\partial Q}{\partial L}=\frac{w}{P}
$$

On a donc :

$$
Q_{0}=F\left(\dot{K}_{0}, L_{0}\right)
$$

désormais considéré comme une donnée. La demande effective s'écrit :

$$
D=C\left(Q, i, \frac{A}{P}\right)+I\left(K_{0}, \mathcal{Q}, i\right)
$$


c'est’à-dire :

- la consommation $C$, une fonction du revenu national $(Q)$, du taux d'intérêt $(i)$ et de la richesse réelle $\frac{A}{P}=a, A$ étant la ri chesse nominale dont on précisera le sens plus loin;

- et l'investissement $I$, fonction de l'output, du taux d'intérêt et du stock de capital accumulé dans le passé considéré comme un paramètre à court terme. $\mathrm{La}$ relation (4) peut se mettre sous la forme agrégée:

$$
D=D\left(Q, i, \frac{A}{P}\right)
$$

On peut préciser déjà la nature des relations (4) et (5), soit :

a)

$$
\frac{\partial C}{\partial Q}>0 \quad \frac{\partial C}{\partial_{i}}<0 \frac{\partial C}{\partial\left(\frac{A}{P}\right)}>0
$$

$$
\frac{\partial I}{\partial K}<0 \quad \frac{\partial I}{\partial Q}>0 . \quad \frac{\partial I}{\partial i}<0
$$

b)

$$
\frac{\partial D}{\partial Q}>0 \quad \frac{\partial D}{\partial_{i}}<0 \frac{\partial D}{\partial\left(\frac{A}{P}\right)}>0
$$

Une hausse de l'output stimule la consommation (propension mar. ginale à consommer positive) et l'investissement (principe de l'ac' célérateur ou prévisions favorables de la part des entrepreneurs) ; la hausse du taux d'intérêt réduit les investissements (l'efficacité marginale nette de l'investissement étant réduite) de même qu'elle augmente l'épargne (ceci n'est pas strictement requis pour notre démonstration et reste discutable ; cette hypothèse n'est pas en fait capitale, du moment que $\frac{\partial D}{\partial i}<0$ via l'effet du taux d'intérêt sur l'investissement).

Enfin, l'accumulation du capital tend à réduire son propre taux d'augmentation et on note la présence de l'effet Pigou ; cependant, dans la relation (5), si $K$ n'était pas constant, l'effet de la richesse 
réelle pourrait devenir négatif à cause de la «décumulation » du capital.

L'équilibre monétaire requiert:

$$
L(Q, P, i)=\bar{M}
$$

où $M_{s}=\bar{M}$ est l'offre de monnaie considérée comme une donnée et $L=L(Q, P, i)$ est la demande de monnaie avec les hypothèses habituelles :

$$
\frac{\partial L}{\partial Q}>0 \quad \frac{\partial L}{\partial P}>0 \quad \frac{\partial L}{\partial i}<0
$$

On pourrait adjoindre en plus une équation décrivant l'équilibre sur le marché des obligations [6] mais grâce à la loi de Walras, on peut ignorer ce marché et concentrer notre attention sur (5) et (6). Ceci, remarquons-le, est un choix arbitraire et les résultats que nous allons obtenir seraient les mêmes si nous ouvrions avec (5) et le marché des obligations (ignorant [6]).

L'output de plein emploi étant toujours assuré par le parfait fonctionnement du marché du travail, il vient :

$$
\therefore D\left(Q_{0}, i, \frac{A}{P}\right)=Q_{0}
$$

Ce qui est l'équilibre sur le marché de l'output de l'économie entière (que l'on considérera comme fermée pour les fins de la démonstration).

A partir du système représenté par (7) et (6), on peut écrire :

$$
\begin{aligned}
& \frac{\partial D}{\partial i} \frac{d i}{d M}+\frac{\partial Q}{\partial\left(\frac{A}{P}\right)} \frac{d\left(\frac{A}{P}\right)}{d M}=0 \\
& \quad \frac{\partial L}{\partial i} \frac{d i}{d M}+\frac{\partial L}{\partial P} \frac{d P}{d M}=1
\end{aligned}
$$


Et, par conséquent, en faisant apparaittre les élasticités convenables :

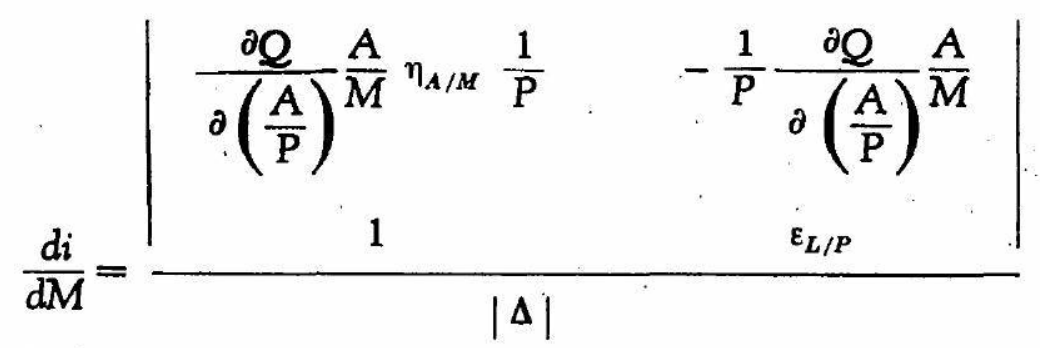

où évidemment :

$$
|\Delta|=\left|\begin{array}{cc}
\frac{\partial Q}{\partial i} & -\frac{1}{P} \frac{\partial Q}{\partial\left(\frac{A}{P}\right)} \frac{A}{M} \\
\frac{\partial L}{\partial i} & \varepsilon_{L / P}
\end{array}\right|
$$

Et :

$$
\eta_{A / M}=\frac{\partial A}{\partial M} \frac{M}{A}
$$

et :

$$
\varepsilon_{L / P}=\frac{\partial L}{\partial P} \frac{P}{L} .
$$

Et de là, on en déduit facilement :

$$
\frac{d i}{d M}=\frac{\frac{1}{P} \frac{\partial Q}{\partial\left(\frac{A}{P}\right)} \frac{A}{M}\left[1-\varepsilon_{L / P} \eta_{A / M}\right]}{\frac{\partial Q}{\partial i} \varepsilon_{L / P}+\frac{1}{P} \frac{\partial Q}{\partial\left(\frac{A}{P}\right)} \frac{A}{M} \frac{\partial L}{\partial i}}
$$

Il s'agit maintenant de discuter l'expression (8) ${ }^{2}$.

D'äbord le cas où il n'existe aucun effet de richesse: toute la monnaie qui circule dans le système économique est uniquement

2. Cette démonstration a été formulée par le professeur G.R. Sparks dans un de ses cours de théorie monétaire au Massachusetts Institute of Technology. 
de la monnaie endogène (pour les concepts de monnaie exogène (outside money) et monnaie endogène (inside money), voir [1]) et sous l'hypothèse de l'absence d'effet de répartition, la monnaie n'apporte aucun effet de richesse. Si les prix nominaux doublent, les pertes infligées aux créanciers voient leurs conséquences neutralisées par les gains des débiteurs. De plus, on suppose dans ce cas qu'il n'existe aucune dette de l'Etat: tous les titres en circulation proviennent de source privée; le stock de capital est aussi fixe à cet instant. On a donc comme résultat, si $A=0$ :

$$
\frac{d i}{d M}=0
$$

et la monnaie est par conséquent neutre. Comme l'équation (7) se ramène à $D\left(Q_{0}, i\right)=Q_{0}$, le taux d'intérêt est déterminé dans le secteur réel: on retrouve donc la dichotomie entre le secteur réel et monétaire et l'équation monétaire (6) sert à déterminer le niveau des prix. Cependant, une remarque très importante s'im. pose ici $[5$, p. 84] : même si l'effet de richesse disparaît pour l'économie entière et que la dichotomie entre le secteur réel et monétaire existe, cela ne signifie pas que les excès des demandes des individus dans l'économie obéissent au postulat d'homogénéité et soient in. changés par suite d'une variation équi-proportionnelle dans les prix. Tout se passe comme si le postulat d'homogénéité jouait mais ceci n'est qu'une illusion macróéconomique résultant de l'agrégation des demandes individuelles: chacune de ces demandes reste sensible aux fluctuations des prix monétaires grâce à l'effet des encaisses réelles, mais l'effet net pour l'ensemble de l'économie où aucune dette publique n'apparaît (monnaie ou autre) est nul. D'où le premier théorème :

«S'il n'y a aucune dette publique (monnaie ou titre) et que la monnaie en circulation est purement endogène, la monnaie est neutre dans un système où il existe parfaite flexibilité des prix et la dichotomie entre le secteur réel et monétaire existe même sans la présence du postulat d'homogénéité. 》

Et on en déduit les résultats suivants :

a) le taux d'intérêt assure l'égalité entre l'offre de plein emploi et la demande globale via son effet sur l'investissement et l'allo cation intertemporelle de la consommation ; 
b) le niveau des prix est déterminé de façon unique par le marché monétaire :

$$
L\left(Q_{0}, i_{0}, P\right)=\bar{M}
$$

$i_{0}$ étant la solution de (7), un certain $P=P_{0}$ assure l'équilibre sur ce marché et toute variation dans $M_{0}$ entraîne un changement dans $P$. La forme particulière de $L$ dépend des habitudes de transaction et des préférences des individus quant à la gestion de leur portefeuille. La dichotomie n'empêche pas le niveau des prix d'être dée terminé de façon «valide» (pour employer le langage de Patinkin), c'est’à-dire sans contradiction intrinsèque dans le système économique.

C'est cette distinction entre les excès de demandes individuelles reposant sur l'actif financier des ménages (effet des encaisses réelles et le choix optimal d'un portefeuille) et la disparition de l'effet de richesse au niveau macróéconomique qui semble avoir échappé aux auteurs Gurley et Shaw [1, chap. V] lesquels se refusent à faire «l'agrégation nette» des différents bilans des individus de l'économie; or, au plan global, si l'effet de répartition ne joue pas, c'est l'agrégation nette qu'on doit utiliser dans les relations macróéconomiques [8, p. 106]. Et que le niveau des prix soit aussi indéterminé est inexact $[1$, p. 142] comme nous l'indique la relation (9) ou encore la forme particulière adoptée par Pantinkin

$$
L\left(Q_{0}, i\right)=\frac{M_{0}}{P} \text {. }
$$

A long terme, un changement dans la quantité de monnaie endogène laisse les variables réelles inchangées dans ce premier cas $[1$, p. 144]; ceci est juste puisque la monnaie est neutre et le taux d'intérêt nullement influencé par la quantité de monnaie en circulation; mais on décèle quand même dans ce type de modèle un effet de richesse indépendant ${ }^{3}$ de $M$ et qui résulte de l'accumulation du capital ; or cette dernière fait ressentir ses effets à long terme et peut influencer la demande globale et donc le taux d'intérêt. Rappelons que $I=I\left(K_{0}, i, Q_{0}\right)$ à court terme mais que nous écrivons $I(K, Q, i)$ à long terme, d'où l'effet de richesse du capital influençant la demande effective : évidemment, $M$ n'apparaît toutefois

3. Ceci m'a été souligné par le professeur Sparks, au cours d'une discussion. 
pas, même à long terme, comme argument de la demande globale et donc n'influence ni $i$ ni $K$.

On peut maintenant souligner la fragilité de l'hypothèse ayant trait à l'absence d'effet de répartition de la richesse ; c'est probablement ici que Gurley et Shaw auraient trouvé un terrain favo rable à leur critique sur l'agrégation nette, car le comportement des débiteurs face à un niveau des prix doublés n'est pas nécessairement contrebalancé de façon exacte par celui des créanciers. Ce que l'hypothèse nous dit est simple : ce que les débiteurs gagnent, les créanciers le perdent et donc au total, tout s'annule. Or, il peut exister une asymétrie dans les réactions des débiteurs et des créanciers [7]; par exemple, une réduction considérable des prix pourrait amener l'élimination de firmes débitrices, ce qui nous obligerait à faire un nouveau calcul. De plus, les effets dynamiques que l'on ignore en statique affaiblissent la signification de l'hypothèse : l'anticipation des baisses (hausses) subséquentes des prix incite à remettre à plus tard (ou à accélérer) les achats... Toute cette asymétrie de comportement a été discutée à propos de l'effet Pigou ; dans cette circonstance, l'effet de richesse ne disparaît plus, même au niveau global, et notre premier théorème ne peut plus s'appliquer.

Intéressonsnous maintenant au second cas. Si toute la richesse nette se résume à la masse monétaire émise par l'Etat, on a l'identité $A=M$ et donc $\eta_{A / M}=1$. Et nous allons alors distinguer quelques points particuliers pour être en mesure d'évaluer la neutralité de la monnaie :

a) Il y a d'abord l'approche de Patinkin, où $\varepsilon_{L / P}=1$; s'il en est ainsi, soit

et

$$
\eta_{A / M}=1
$$

on trouve:

$$
\varepsilon_{L / P}=1
$$

$$
\frac{d i}{d M}=0
$$

et la monnaie est neutre. Remarquons ici que l'on suppose que seule la monnaie exogène circule dans l'économie et que l'on n'utilise aucune monnaje endogène. Le système se ramène alors à : 


$$
\begin{aligned}
& D\left(Q_{0}, i, \frac{\bar{M}}{P}\right)=Q_{0} \\
& L\left(Q_{0}, i, P\right)=\bar{M}
\end{aligned}
$$

Le taux d'intérêt n'est plus déterminé de façon unique dans le secteur réel mais l'est plutôt par la résolution simultanée du sys' tème; et il en va de même du niveau des prix. Ceci élimine la dichotomie du premier cas. Remarquons que le système précédent, dans le cas spécial où $\varepsilon_{L / P}=1$ peut se mettre sous la forme :

$$
\begin{aligned}
& D\left(Q_{0}, i, \frac{\bar{M}}{P}\right)=Q_{0} \\
& L\left(Q_{0}, i, \frac{\bar{M}}{P}\right)=\frac{\bar{M}}{P}
\end{aligned}
$$

soit la forme utilisée par Patinkin. L'interprétation du système se ramène à la séquence suivante : si on double le niveau des prix, la demande de monnaie double de façon à laisser intacte la valeur réelle de l'encaisse. $\mathrm{La}$ demande de monnaie peut ainsi prendre la forme spécifique :

$$
M_{d}=k\left(P Q_{0}\right)+P L(i)
$$

soit la demande pour les fins de transaction (avec le $k$ traditionnel de Cambridge) et la demande pour les fins de spéculation affectée du niveau des prix. C'est ce que Patinkin appelle l'absence d'illusion monétaire dans la demande de monnaie alors que chez Keynes, les individus ne se soucient pas de la valeur réelle de leur encaisse de spéculation. Cette approche correspond à une reformulation de la théorie quantitative de la monnaie où l'effet des encaisses réelles apparaît comme le mécanisme qui explique comment une augmentation dans $M_{s}$ entraîne une variation à la hausse équi-proportionnelle dans le niveau des prix. Par exemple, si on double $M_{s}$, les individus ont un excès d'encaisse et le taux d'intérêt baisse tempo rairement, ce qui stimule la demande globale et provoque une hausse des prix jusqu'à ce que toutes les relations en termes réels reviennent à leur point initial (c'est-à-dire les prix doublent). Cette notion d'encaisse désirée se retrouve d'ailleurs dans les Eléments de Walras. Enfin, si $M_{d}$ passait de $M_{d}^{*}$ à $M_{d}^{* *}$, ce changement dans la préférence pour la liquidité se faisant au détriment des 
biens et services et des obligations, le taux d'intérêt resterait inchangé [6, pp. 244248].

On a donc un second théorème :

«S'il n'y a aucune dette publique autre que la monnaie émise par l'Etat (monnaie exogène seule) et que les individus se soucient de la valeur réelle de leur encaisse, la monnaie est neutre dans un système où il existe parfaite flexibilité dans les prix et il n'y a pas de dichotomie entre le secteur réel et monétaire. »

Avant d'en terminer avec ce premier aspect, il serait bon d'at' tirer l'attention sur la signification de $\varepsilon_{L / P}=1$. On ne doit pas comprendre par là que chaque individu a une demande de monnaie à élasticité unitaire par rapport aux prix ; lorsqu'on dit que si on double $M_{s}$, on double aussi $P$, ceci décrit un équilibre final et dans l'intérim, c'est-à-dire dans le processus d'ajustement vers l'équilibre, l'élasticité de la demande de monnaie peut changer et c'est la hausse progressive des prix qui nous ramène vers un niveau des encaisses réelles identique à celui du début ; entre temps, les individus peuvent augmenter l'encaisse qu'ils désirent mais la hausse des prix les ramène vers une situation analogue à celle à laquelle ils faisaient face avant cet accroissement dans $M_{s}$ (pour une description systématique de ce mécanisme, voir [1-A]). En d'autres termes, il est important de distinguer l'élasticité de la courbe d'équilibre des marchés qui est $\varepsilon_{L / P}=1$, de l'élasticité de la demande de monnaie de la part des individus dans l'économie.

b) Toujours dans le cadre de l'approche de Patinkin, on peut voir maintenant l'effet de l'introduction de la monnaie endogène. Au premier point de cette section, on a supposé que seule la monnaie exogène circulait dans l'économie. $A$ cette monnaie exogène désignée par $M_{s}^{1}=\bar{M}_{1}$, ajoutons la monnaie endogène $M_{s}^{2}=\vec{M}_{2}$ (dans les deux cas on suppose toujours que chacune des offres est déterminée de façon autonome). Avec $\varepsilon_{L / P .}=1$, on a :

$$
\begin{aligned}
& D\left(Q_{0}, i, \frac{\bar{M}_{1}}{P}\right)=Q_{0} \\
& L\left(Q_{0}, i, \frac{\bar{M}_{1}}{P}\right)=\frac{\bar{M}_{1}+\bar{M}_{2}}{P}
\end{aligned}
$$


On voit très bien que si $M_{s}^{1}$ seule est multipliée par un scalaire $\lambda$, la monnaie n'est plus neutre; car supposons que les prix soient eux aussi multiplés par $\lambda$, on se retrouve devant l'impossibilité d'un taux d'intérêt constant. Si $i=i_{0}$ satisfaisait le système précédent, il ne peut également satisfaire :

$$
\begin{aligned}
& D\left(Q_{0}, i, \frac{\bar{M}_{1}}{P}\right)=Q_{0} \\
& L\left(Q_{0}, i, \frac{\bar{M}_{1}}{P}\right)=\frac{\bar{M}_{1}}{P}+\frac{\bar{M}_{2}}{\lambda P}
\end{aligned}
$$

Par conséquent, si on double $M_{s}^{1}$ seul, les prix et le taux d'inté rêt devront varier de façon à équilibrer le système. En fait, le taux d'intérêt va monter et les prix augmenteront moins que proportionnellement pour assurer l'équilibre. Toutefois, si $M_{*}^{1}$ et $M_{s}^{2}$ sont en même temps doublés, alors la neutralité se vérifie facilement et à l'équilibre on revient au système initial. D'où le troisième théorème :

«S'il n'existe aucune autre dette publique que la monnaie émise par l'Etat et qu'il y ait en même temps de la monnaie endogène, la monnaie n'est pas neutre sauf dans le cas où il y a variation simultanée et dans la même proportion des masses monétaires endogène et exogène dans un système où il $\mathrm{y}$ a parfaite flexibilité des prix et où les individus se préoccupent de la valeur réelle de leur encaisse. 》

Et comme corollaire assez évident : il n'existe pas de dicho tomie entre le secteur réel et monétaire.

c) $\mathrm{Si} \varepsilon_{L / P}<1$, on tombe dans le cas keynésien où la demande de monnaie peut s'écrire :

$$
M_{d}=k\left(P Q_{0}\right)+L(i)
$$

Les agents souffrent d'illusion monétaire quant à l'encaisse qu'ils veulent garder pour les fins de spéculation. Un examen de (8) nous indique que :

$$
\frac{d i}{d M}<0
$$


et donc la monnaie n'est plus neutre. Et ceci vaut aussi pour le cas complexe où se mêlent monnaie exogène et monnaie endogène. D'où une quatrième proposition en se limitant au cas de la monnaie exogène :

«S'il n'existe aucune dette publique autre que la monnaie émise par l'Etat et que les individus ne se soucient pas de la valeur réelle de leur encaisse, la monnaie n'est plus neutre dans un système où il existe parfaite flexibilité dans les prix.»

Le quatrième cas admet, en plus de la monnaie émise par la banque centrale, l'existence d'une dette publique empruntant la forme de titres (désignés par $D$ ) quelconques sur lesquels les autorités monétaires peuvent effectuer certaines opérations (achat ou vente). On $\mathrm{a}^{4}$ :

$$
\frac{A}{P}=\frac{M_{s}+D}{P}
$$

Dans ce qui suit, nous nous limitons au cas de la monnaie exogène seule puisque l'addition de la monnaie endogène ne change rien ici quant au caractère neutre ou non neutre de la monnaie. Evidemment, si une dette publique existe, il vient :

et donc,

$$
\eta_{A / M}<1
$$

$$
\frac{d i}{d M}<0
$$

Et même dans le cas où $\varepsilon_{L / P}=1$, la monnaie n'est plus neutre et ce, à cause d'un équilibre de portefeuille devenu plus complexe. Désormais, les ménages n'ont plus simplement une encaisse désirée mais plutôt un rapport désiré entre les titres et la monnaie. Ce rapport peut être fonction du taux d'intérêt tel que si $i$ est élevé, on préférera avoir plus de titres dans son protefeuille. Il n'est donc pas possible de faire un partage dans la richesse nette que la société détient; cette composition de la dette changera selon les opéra-

4. Nous avons écrit $\frac{M_{0}+D}{P}$ mais en fait, si le public actualise les taxes qu'il aura à payer pour financer les intérêts à verser de la même façon qu'il actualise les intérêts reçus, l'effet de richesse de $D$ disparaitra. D'où on peut écrire $M+d K$ où $d$ est un facteur qui représente jusqu'à quel point ce processus d'actualisation n'est pas fait. Pour des raisons de simplicité on pose $D=1$ ce qui n'affecte pas le résultat final mais laisse sous-entendre qu'aucune actualisation n'est faite. $[6, p .289]$. 
tions des autorités monétaires. Si ces dernières veulent accroître $M_{s}$, elles devront abaisser le taux d'intérêt de façon à persuader les individus de détenir plus de monnaie et de réaliser des gains de capital sur les titres qu'ils possèdent. Le système se ramène alors à :

$$
\begin{gathered}
D\left(Q_{0}, i, \frac{\bar{M}+\bar{D}}{P}\right)=Q_{0} \\
L\left(Q_{0}, i, P\right)=\bar{M}
\end{gathered}
$$

et donc $P$ et $i$ sont déterminés par la résolution simultanée des deux équations. D'où le dernier théorème (qui vaut d'ailleurs quelle que soit la valeur de $\varepsilon_{L / P}$ ) :

«S'il existe une dette publique complexe (monnaie et titres), toute variation dans l'offre de monnaie, c'est-à-dire émission pure de monnaie ou achat et vente de titres en open market, va faire varier le taux d'intérêt pour assurer un équilibre de portefeuille pour l'ensemble de la société et donc la monnaie n'est pas neutre. 》

A la vérité, une exception accompagne ce théorème. En effet, (toujours en supposant l'absence de monnaie endogène), si $\varepsilon_{L / P}=1$ et que les titres émis par l'Etat possèdent une clause escalatrice, une augmentation dans $M_{8}$ fera augmenter tous les prix de façon équi-proportionnelle, y compris le prix des titres et, par conséquent, laissera inchangée la valeur réelle des titres détenus en portefeuille et donc le taux d'intérêt n'aura pas à changer. Avec $\varepsilon_{L / P}=1$, on peut utiliser le système de Patinkin pour vérifier cette proposition, en supposant l'existence d'obligations ayant leur valeur rattachée au niveau des prix :

$$
\begin{aligned}
& D\left(Q_{0}, i, \frac{\bar{M}+P \bar{D}}{P}\right)=Q_{0} \\
& L\left(Q_{0}, i, \frac{\bar{M}+P \bar{D}}{P}\right)=\frac{\bar{M}}{P}
\end{aligned}
$$

Si $i=i_{0}$ vérifie ce système (avec $P=P_{0}$ comme seconde racine), il vérifie aussi le système où $\bar{M}$ et $P$ ont été multipliés par $\lambda$ puisque le scalaire apparaît au numérateur et au dénominateur; d'où la neutralité de la monnaie. Mais cette neutralité implique une opé 
ration bien spécifique de la banque centrale, à sávoir, l'augmentation de l'offre de monnaie exogène sans apporter aucune modification au volume des titres d'Etat en circulation. Si on augmente $M_{s}$ en achetant des titres et non en créant simplement de la monnaie, on change alors la composition du portefeuille des individus et le taux d'intérêt devra varier. Ceci se vérifie aisément : soit un achat qui réduit de $\lambda$ le volume des titres en circulation et qui augmente d'autant le stock de monnaie; la masse monétaire devient $(1+\lambda) M$ et supposons que le niveau des prix (avec $\varepsilon_{L / P}=1$ ) soit égal à $(1+\lambda) P_{0},\left(P=P_{0}\right.$ étant le niveau des prix qui satisfait notre dernier système). On voit immédiatement que l'ancien taux $i=i_{0}$ ne peut plus satisfaire le système qui se ramènerait $\grave{a}$ :

$$
\begin{aligned}
& D\left(Q_{0}, i^{*}, \frac{\bar{M}}{P_{0}}+\frac{(1-\lambda) D}{(1+\lambda)}\right)=Q_{0} \\
& L\left(Q_{0}, i^{*}, \frac{\bar{M}}{P_{0}}+\frac{(1-\lambda) D}{(1+\lambda)}\right)=\frac{\bar{M}}{P_{0}}
\end{aligned}
$$

où $i^{*}$ deviendrait le nouveau taux à trouver. En fait, il est bien évident que le taux d'intérêt et le niveau des prix devront simultanément s'ajuster de façon à satisfaire le nouveau système (dans le modèle suggéré ici, les prix vont augmenter plus que dans la proportion $\lambda$ et le taux d'intérêt baissera). Par conséquent, une opération d'open market modifie la valeur réelle des titres détenus en portefeuille (en changeant la quantité des titres) et entraîne nécessairement un changement dans $i$; la monnaie perd sa neu tralité. Cette nuance apportée au dernier théorème avait été étudiée par L. Metzler dans un article fondamental sur cette question [5-A]; Metzler n'avait pas utilisé l'hypothèse d'obligations à clause escalatrice mais avait supposé la présence d'actions ordinaires dont le prix variait de la même manière que le niveau des prix et sur lesquelles la banque centrale faisait ses opérations d'open market.

Terminons cette première partie par quelques mots sur l'introduction de la rigidité des prix ou des salaires. On tombe alors dans un univers bien keynésien où l'influence de la politique monétaire s'exerce par les effets des variations de $M_{s}$ sur le taux d'intérêt. L'accroissement de l'offre de monnaie n'affecte pas en soi les prix et l'emploi; le taux d'intérêt devient alors le mécanisme par lequel les 
changements dans $M_{s}$ peuvent influencer les prix, l'emploi et la production et on ne saurait alors parler de neutralité. Au plein emploi, Keynes [4] admet en première approximation la théorie quantitative ; or, sa formulation, à cause de l'illusion monétaire qu'elle implique (telle que discutée plus haut) amène toujours (sauf trappe de liquidité) une variation de $i$ quand $M_{8}$ change et là encore, on ne peut conclure à la neutralité de la monnaie comme le supposerait la théorie quantitative pure avec $\varepsilon_{L / P}=1$.

Alors qu'en statique la question de la neutralité se résume à l'absence d'influence sur le secteur réel d'une variation donnée et finale de la masse monétaire, en dynamique, il s'agit d'examiner les effets d'un changement dans le taux d'expansion de $M$ sur le sentier de croissance décrit par une économie. En statique, on se pose la question suivante: qu'advient-il des prix et du taux d'intérêt et donc de l'emploi et de la production lorsqu'on double le stock de monnaie qui existe dans une économie. En dynamique, on s'interroge sur les effets d'un changement du taux d'expansion $0_{1}=\left(\frac{\dot{M}}{M}\right)$ (où $\dot{M}=\frac{d M}{d t}, t$ étant la variable «temps »), au taux $\theta_{2} \geqslant \theta_{1}$. Par exemple, un taux $\theta_{1}$ entraînet til une formation plus élevée de capital au cours du temps qu'au taux $\theta_{2}$ ? C'est à ces questions que l'analyse dynamique s'intéresse.

Dans le modèle courant à un secteur avec une fonction de production macróéconomique linéaire et homogène $[10,11]$, rien n'empêche la formation du capital d'être poussée à un point tel que le taux de rendement sur le capital devienne négatif. Dans ces modèles où il n'existe qu'une forme d'accumulation de la richesse (soit le capital), et où la décision d'investir ou d'épargner n'est pas distincte, l'économie peut atteindre un sentier de croissance naturelle où le taux de rendement sur $K$ est très faible sans qu'il n'y ait aucun problème de demande effective.

Soit la fonction traditionnelle (qui satisfait les conditions d'Inada), 


\section{LACTUALITE ECONOMIQUE}

$$
Q=F(K, L)
$$

qui s'écrit en ramenant le tout per capita (on peut ignorer le pro grès technique ou supposer qu'il est neutre de type harrodien et alors définir toutes les variables en termes «d'unité d'efficacité ») :

$$
q=f(k)
$$

et où le taux de rendement sur $K$ est :

$$
\frac{\partial F}{\partial K}=f^{\prime}(k)=\rho
$$

La fonction d'épargne $S=s Q$ et le taux $\frac{\dot{L}}{L}=n$ (croissance naturelle de la main-d'œuvre) nous permettent d'écrire l'égalité traditionnelle entre le taux garanti et le taux naturel (avec $v=K / Q)$ :

$$
\frac{s}{k / q}=\frac{\dot{L}}{L} \text { ou } \frac{s}{v}=n
$$

Or, à ce niveau, il est possible que $\rho<0$. Si tel est le cas, $s$ peut diminuer et la réduction de l'épargne s'accompagne d'une augmentation correspondante de $C$ et donc l'équilibre au plein emploi continue d'exister.

Si on introduit l'hypothèse de Harrod, soit d'un taux de rendement minimum $\rho$ et qu'à ce taux, le volume d'épargne permette encore un deepening du capital $\left(G_{w}>G_{n}\right)$, les difficultés keyné siennes apparaissent: les investisseurs n'entreprendront pas une quantité suffisante d'investissements pour éponger le volume de l'épargne. Mais un taux de rendement minimal n'existe pas dans le vide : il doit refléter la concurrence des autres formes d'accumulation de la richesse. Il devient donc nécessaire de réintroduire la monnaie dans le modèle à titre d'actif alternatif à $K$ (et évidemment il s'agit de monnaie purement exogène). Cette introduction de la monnaie et les changements qu'elle apporte dans l'analyse de la croissance font essentiellement l'objet du récent article publié par le professeur Tobin $[12,13]$.

En introduisant la monnaie comme actif alternatif, il est néces saire de préciser le comportement des agents économiques dans la 
gestion de leur portefeuille. Retenons deux cas parmi ceux qui sont proposés par Tobin, soit le plus simple et le plus complexe.

Si le niveau des prix est fixe, la décision d'accumuler $M$ ou $K$ dépendra du rendement relatif de $M$ et de $K$. Comme l'Etat est en mesure de fixer le rendement sur $M$, il contrôle également $\rho$ en supposant par exemple que les investisseurs préfèrent une forme de richesse à l'autre dès que son rendement est plus élevé (on ne détient que $K$ dans son portefeuille si $\rho>i$, $i$ étant le taux de rendement propre à la monnaie). On peut voir alors comment une politique monétaire et fiscale adéquate élimine l'impasse keynésienne dont nous parlions. Si le taux garanti excède le taux naturel au niveau de $\frac{\partial F}{\partial K}=\bar{\rho}$, un widening du capital implique :

$$
n k+(1-s) f(k)<f(k)
$$

c'est-à-dire une insuffisance de la demande globale. Si l'État désire combler cette déficience de la demande, il peut y parvenir en encourant un déficit qui absorberait l'excès d'épargne. Soit alors $\frac{\dot{M}}{Q}$ l'émission nouvelle de monnaie par unité d'output; le revenu disponible devient ainsi :

$$
f(k)+\frac{\dot{M}}{Q} \frac{Q}{L} \quad \text { ou } \quad f(k)+d f(k)
$$

où $d=\frac{M}{Q}$ est le déficit par unité d'output.

A l'équilibre:

$$
(1-s) f(k)(1-d)+n k=f(k)
$$

D'où l'on tire :

$$
d=\frac{s-g v}{1-s}
$$

L'expression (6) indique exactement le déficit (émission de monnaie) nécessaire pour éliminer l'impasse keynésienne.

Le second cas est plus intéressant; si $P$ est variable et que les investisseurs choisissent $K$ ou $M$ selon leur préférence et en fonc- 


\section{L'ACTUALITE ECONOMIQUE}

tion de leur rendement respectif tel que si $\rho>i$ ils accumulent davantage de capital et moins de monnaie, l'émission de monnaie a une influence plus profonde sur l'économie. En effet, le rende' ment de la partie monétaire de la richesse a un double aspect : le taux de rendement propre à $M$ (qu'on a désigné par $i$ et fixé nominalement par l'Etat) et aussi le taux de changement dans la valeur réelle de $M$ (gains de capital dont bénéficient les investis. seurs si la croissance de l'output est supérieure à $\dot{M}$ ). D'où les gains de capital servent à augmenter le revenu disponible et jouent donc le même rôle que le déficit du premier cas. Mais il y a plus dans le second cas : à l'équilibre (croissance soutenue où toutes les variables de l'économie ont un taux d'expansion analogue), le stock réel de monnaie croît au même rythme que le capital, soit $n$. Si l'Etat laisse $\frac{M}{P}$ croître par le jeu de $\frac{\dot{P}}{P}<0\left(\frac{\dot{P}}{P}\right.$ étant aussi égal à $n$ en valeur absolue), ceci implique un taux de rendement plus élevé sur la monnaie que si $\frac{\dot{\mathrm{P}}}{\mathrm{P}}>0$ et donc une préférence accrue pour la monnaie dans le portefeuille des investisseurs. De là, la nonneutralité de la monnaie; si l'État augmente le taux d'expansion de $M$, il en résultera une hausse du taux d'accroissement des prix et donc des pertes de capital et une réallocation de la richesse dans le portefeuille des individus : ils accumulent plus de capital puisque son rendement relatif s'est accru et réduiront leur stock de monnaie sur lequel ils ont subi des pertes de capital. (Remarquons qu'après l'accroissement du taux d'émission de $M$, le revenu disponible augmente et la consommation va aussi aug. menter comme premier effet avant que les prix aient commencé à monter; et donc $S$ diminue et $\dot{K}$ également. Mais ceci est temporaire et le mouvement sera renversé par la hausse des prix.) Tobin conclut donc à l'effet favorable d'un haut taux d'expansion de $M$ sur la formation du capital.

On a soutenu que l'approche de Tobin ne reposait pas sur un comportement d'optimisation des individus [9]. Une seconde façon d'aborder le problème consiste à supposer que les différents agents analogues d'une économie (on s'intéresse à l'homme représentatif) ont une fonction de préférence invariable dans le temps qui dépend 
de leur consommation (en termes réels au temps $t, c(t)$ ) et du stock de monnaie $m(t)$ en termes réels qu'ils détiennent (l'utilité dépendant du flux de biens et services que l'on peut se procurer avec cette quantité de monnaie). Toute la question revient alors à maximiser la fonctionnelle :

$$
\operatorname{Max} V=\int_{0}^{\infty} U(c(t), m(t)) e^{-\delta t} d t
$$

où $\delta$ est le taux de préférence temporelle des individus considéré comme une constante dans le temps. $A$ cette fonctionnelle, s'ajoutent une contrainte de flux (plein emploi : tout le revenu produit est utilisé) et une contrainte de stock $(a(t)=k(t)+m(t))$. Ce qui se ramène à un problème de calcul des variations et donc à dériver les équations d'Euler et les conditions jacobiennes.

Telle est l'idée essentielle de ce modèle; tel que formulé, la monnaie est neutre: si on double $\frac{\dot{M}}{M}$, on double $\frac{\dot{P}}{\mathrm{P}}$ au niveau de la croissance soutenue puisque de toute façon l'économie aboutit avec un stock de capital défini par le taux de préférence temporelle

$$
\frac{\partial F}{\partial K}=\delta
$$

Comme $\delta$ est constant, l'accumulation du capital n'est pas affectée par un changement dans le taux d'expansion de $M$. (Mais le $\delta$ constant n'est pas essentiellement la cause de cette neutralité; la neutralité exige la formulation adoptée par l'auteur car si on pose $U=U(c(t), a(t))$ où $a(t)=k(t)+m(t)$ et que $\delta$ soit constant, la monnaie n'est plus neutre). Comme $M$ entre dans $U$, il est pos' sible de dériver un taux d'expansion optimal de $M$ dans le sens où, compte tenu du stock de capital dérivé par $\frac{\partial F}{\partial K}=\delta$, la fonc tion $U$ soit maximisée.

Ce modèle utilise seulement de la monnaie exogène comme celui de Tobin. Il repose également sur l'hypothèse discutable [3] que le taux de préférence temporelle est constant ; une approche plus réaliste mais plus complexe serait de poser $\delta(t)=f(a(t))$, c'est- 


\title{
L'ACTUALITE ECONOMIQUE
}

à-dire de supposer une dépendance de $\delta$ au cours du temps du niveau de richesse accumulée .

Plusieurs points n'ont pas encore été touchés dans le domaine de la dynamique; par exemple, l'utilisation des modèles où la monnaie est endogène seulement; ou encore endogène et exogène à la fois. De plus, le développement de la théorie de la croissance (modèles pluri-sectoriels, entre autres), pourra fournir de nouveaux modèles où il sera intéressant de voir les modifications ap. portées par l'introduction de la monnaie.

\author{
Yves RABEAU, \\ chargé d'enseignement à l'École \\ des Hautes Etudes commerciales (Montréal).
}

\section{BIBLIOGRAPHIE}

1-A. Archibald G.C. et Lipsey R.G., «Monetary and Value Theory : a Critique of Lange and Patinkin », Review of Economic Studies, octobre 1958.

1. Gurley J.G. et Shaw E.S., Money in a Theory of Finance, The Brookings Institute, 1960.

2. Johnson H.G., « Monetary Theory and Policy », American Economic Review, juin, 1962.

3.

«The Neo-Classical one Sector Growth Model, a Geometrical Exposition and Extension to a Monetary Economy 》, Economica, août 1966.

4. Keynes, J.M., La théorie générale de l'emploi, de l'intérêt et de la monnaie », Payot, Paris, 1963.

5-A. Metzler, L.A., «Wealth, Saving and the Rate of Interest », Joumal of Political Economy, avril 1951.

5. Nous nous proposons dans un autre article d'examiner plus en détail les effets de la monnaie sur la croissance économique et de montrer comment la théorie de la croissance optimale (basée sur le calcul des variations et sur le principe de Pontrya. gin) est modifiée par l'introduction de la monnaie. 
5. Modigliani, F., «The Monetary Mechanism and its Interaction with Real Phenomena », Review of Economics and Statistics, février 1963.

6. Patinkin, D., Money, Interest and Prices, Harper and Row, 2e édition, 1965.

7. ........................, «Price Flexibility and Full Employment, American Economic Review, septembre 1948.

8. ........................., « Financial Intermediaries and the Logical Structure of Monetary Theory 》, American Economic Review, 1961.

9. Sidrauski, M., « A Theory of Economic Growth in a Monetary Economy », article non publié, MIT, 1966-67.

10. Solow, R.M., «A Contribution on the Theory of Economic Growth », Quarterly Joumal of Economics, février 1956.

11. Swan, T.W., «Economic Growth and Capital Accumulation 》, Economic Record, novembre 1956.

12. Tobin, J., «A Dynamic Aggregative Model », Journal of Political Economy, avril 1955.

nometrica, octobre 1965. 